\title{
Nanooncology: Perspective on Promising Anti-Tumor Therapies
}

\author{
Alain L Fymat* \\ International Institute of Medicine and Science, USA
}

Submission: April 01, 2017; Published: April 24, 2017

*Corresponding author: Alain L Fymat, International Institute of Medicine and Science, California, USA, Tel: (760) 485-9149;

Email: alain.fymat@fiimas.org

\section{Abstract}

The long quest for cancer cures started approximately 4,000 years ago, but the modern "war on cancer" was waged only during the past five decades. While considerable progress has since been made in a number of cancer types, a lot remains to be done. Progress had been slowed owing to our lack of deep understanding of cell biology and the true nature of the disease. We now understand that cancer is both genetically and environmentally caused. On the genetic side, the disease, a clonally evolving one, is caused by mutations that are unleashed during abnormal cell growth. With the advent of synthetic biology and genomics, great advances in our understanding of cancer have been and continue to be made. Its hallmarks will be provided as well as the therapeutic empirical principles that have been evidenced in several of the cancer treatment modalities (chemotherapy, radiation therapy, surgery, hormone therapy, natural and synthetic immunotherapy, a combination of some of the above approaches). The novel modality of nanooncology will be discussed at length as it provides a new vista on the subject. The underlying nanotechnlogy and its applications in medicine, in general, and in oncology, in particular will be reviewed. Of special interest are the applications of nanoparticles against cancer such as in vivo imaging, drug delivery (including delivery across and around the blood brain barrier), the detection of faint early traces of cancer, and others. Recent advances in the field will be covered and a tentative comparison given between traditional chemotherapy and nanochemotherapy. Treatments of various types of malignant tumors will also be covered (of the brain, lungs, pancreas, eyes and others). Joining other cancer research scientists, I will outline the new directions urged to improve cancer nanotechnology.

Abbreviations: BBB: Blood Brain Barrier; CAR: Chimeric Antigen Receptors; CPMV: CowPox Mosaic Virus; ER: Estrogen Receptor; FQD: Fluorescent Quantum Dots; GBM: GlioBlastoma Multiform; GF: Growth Factor; GIS: Growth Inhibitory Signal; 3HM: Helix Micelle; IR: InfraRed (light); MNP: Magnetic Nano Particle; MRI: Magnetic Resonance Imaging; ND: Nanoscale Device; NI: Nano Imaging (Tracer) Agent; NIR: Near Infra-Red (light); NM: Nano Medicine; NO: Nano Oncology; NP: Nano Particle; NT: Nano Technology; PD-1: Programmed Death-1; PDT: Photo Dynamic Therapy; PET: Positron Emission Tomography; QD: Quantum Dots; RCT: Rational Combination Therapy; RF: Radio-Frequency; T-MOC: Tumor Microenvironment On-Chip; TPT: Targeted Photothermal Therapy; TS: Tumor Suppressor; UV: Ultra-Violet (light)

\section{Introduction and Background}

Cancer is possibly the oldest disease seen in human specimens and the quest to battle it began some 4,000 years ago. However, hidden by age and other diseases (tuberculosis, dropsy, cholera, smallpox, leprosy, plague and pneumonia), it was fleetingly rare. As these other diseases were vanquished, and as the human lifespan lengthens, cancer emerged [1].

Many diseases are lumped together under the denomination "cancer" because they share the fundamental biological feature of abnormal cell growth unleashed by mutations. Mutations occasionally occur as the cells divide, affecting cell behavior and sometimes causing more frequent growth and division. Normally, cell division responds to growth factors (GF) and stops when encountering growth inhibitory signals (GIS) from surrounding cells. After a number of divisions, the cell dies and remains within the epithelium from which it is unable to migrate to other organs. To become cancerous, it would have to bypass these signals and accumulate new genetic mutations in a number of genes, the most frequent being a loss of function of the p53 protein-a tumor suppressor (TS) or in the p5 pathway and/or a gain of function through mutations in the protein or in other oncogenes. It will then keep growing, escape from the epithelium and the primary tumor, cross the endothelium of a blood vessel, be transported by the blood stream and colonize (a) new organ(s) to give rise to metastases. A cancer cell is thus an astonishing perversion of the normal cell whilst, like it, it relies on growth in the most basic elemental sense. By contrast, a cancer cell grows until every visible sign of it had vanished and it divides frenetically without a regulating mechanism-a clonal process. However, cancer is more than a clonal disease, it is a 
"clonally evolving" disease. If growth occurred without evolution, cancer cells would not have the capacity to invade, survive, and metastasize. Every generation of cancer cells is partly different from its genetic parent. When mutating, it can resist attack by a chemotherapeutic drug or by the autoimmune system; it can generate new cells that are increasingly adapted to survival and growth, and can even speed up other mutations. The fittest cancer cells survive - the ultimate product of Darwinian selection of the fittest. One would naively think that cancer could be defeated by either preventing mutations from occurring in normal cells or, else, finding the means to eliminate the mutated cells without compromising normal growth. Unfortunately, cancer is woven into our genome, the mutated genes are but distorted versions of the normal ones, and both are braided together [2-4]. Unbraiding them continues to be the most formidable undertaking in clinical medicine.

In developing cancer, individuals differ in both their inherited tendency (the genetics) and exposure to the environment (the epigenetics and ecogenetics) [5-10]. The vast majority of cancer cases are due to environmental risk factors, many of which (but not all) being controllable lifestyle choices and thus preventable. It has been suggested that more than $30 \%$ of cancer deaths could be prevented by avoiding risk factors including: tobacco, overweight, obesity, insufficient or/ and inappropriate diet, physical inactivity, alcohol, transmitted infections, and air pollution. However, not all environmental causes are controllable such as naturally occurring background electromagnetic radiation which, fortunately, is a comparatively much smaller factor.

\section{The hallmarks of cancer}

Research about cancer genetic causes has focused on the following issues:

a) What are the agents (e.g. viruses) and events (e.g. mutations) that cause or facilitate genetic changes in cells destined to become cancerous?

b) What is the precise nature of the genetic damage, and the genes that are affected by it?

c) What are the consequences of those genetic changes on the biology of the cell, both in (a) generating the defining properties of a cancer cell, and (b) facilitating additional genetic events that lead to further progression of the cancer.

As a result of the above, six hallmarks of cancer have been set forth [11]:

i. Self-sufficiency in growth signals: Cancer cells acquire an autonomous drive to proliferate-pathological mitosis- by virtue of the activation of oncogenes such as ras (contained in a fragment of DNA present in all cells) and myc (the first engineered gene in a mouse).

ii. Insensitivity to growth inhibitory (antigrowth) signals: Cancer cells inactivate tumor suppressor genes (such as retinoblastoma, $\mathrm{Rb}$ ) that normally inhibit growth. iii. Evasion of programmed cell death (apoptosis): Cancer cells inactivate and suppress genes and pathways that normally enable cells to die.

iv. Limitless replicative potential: Cancer cells activate specific gene pathways that render them immortal even after generations of growth.

v. Sustained angiogenesis: Cancer cells acquire the capacity to draw out their own supply of blood and blood vessels (tumor angiogenesis).

vi. Tissue invasion and metastasis: Cancer cells acquire the capacity to migrate to other organs, invade other tissues, and colonize these organs, resulting in their spread throughout the body.

\section{Empirical principles of cancer therapy}

In the course of cancer treatments, several empirical principles have emerged. These principles have been categorized below as they relate to specific therapeutic modes:

\section{General principles [12]:}

a. Prevention is the best cancer treatment.

b. Cancer is an organized chromosomal chaos: Specific and identical mutations exist in particular forms of cancer.

c. Enormous genetic and anatomic heterogeneity. Understanding that heterogeneity is of deep consequence, having led to the meticulous separation into distinct stages and into ER (estrogen receptor)-positive and -negative that require different treatment approaches.

d. Physiological memory retention of the non-cancerous origin: This is the case of, for example, the thyroid and the prostate.

e. Attacking a cancer cell specifically begins by identifying its biological behavior, genetic makeup, and unique vulnerabilities.

f. Meticulous matching of each particular form and stage of cancer to a particular therapy.

g. Early stage local cancers are often inherently different from widely spread metastatic cancers even within the same form of cancer.

h. Tumor potential for metastasizing is more important than its presence and size: Importance of identifying the underlying mutations, gene locations, and mechanism(s).

i. Continued treatment even long after the cancer has visibly vanished.

\section{In chemotherapy [13-18]:}

I. Sensitivity to certain chemicals: Cancer cells' unique and specific vulnerabilities render them particularly sensitive to certain chemicals that may have little impact on normal cells. 
II. The ideal chemical anti-cancer drug identifies a specific molecular target and attacks it.

III. Indiscriminate chemotherapy may not be the only strategy by which to attack cancer.

IV. Toxicities notwithstanding, annihilating cancer might involve a synergistic multiple drug chemotherapy: Different drugs elicit different resistance mechanisms, and produce different toxicities. Their combination dramatically lowers the chance of resistance and increases cell killing.

V. Chemotherapeutic treatment is an iterative process: Chemotherapy typically kills only a fixed percentage of cancer cells at any given instance.

\section{In radiation therapy [13]:}

a. A palliative or control, rarely curative.

b. More effective for localized tumors: More discriminating therapy is needed for non-localized cancers.

c. Limited use for metastatic cancers.

d. Induces cancer: X-rays kill rapidly dividing cells (DNA damage) and create cancer-causing mutations.

e. Synergism with surgery after chemotherapy.

\section{In surgery [13]:}

1. Immediate success is not a predictor of long-term success: Including the ability to decrease the relapse of cancer.

2. Ultimate survival depends on how extensive the metastasis.

3. Synergism after chemotherapy and/or radiation therapy.

\section{In combined multi-therapeutic modes [19]:}

i. After chemotherapy and surgery, use adjuvant chemotherapy to decrease elapses.

\section{In hormone therapy:}

A. Sustained growth and propagation by the host's hormonal function: Cancer is not necessarily autonomous and self-perpetuating.

B. Temporary prolonged remissions followed by relapses.

\section{In immunotherapy [20-24]:}

a) The immune system can subvert the cancer treatment.

b) Natural immune therapies include: DNA origami/trojan horse, conversion of the binary form of the enzyme mnk-2 to overcome drug resistance, and Programmed Death (PD)-1 inhibitors.

c) The DNA origami technique should work on most any form of drug-resistant cancer. d) Conversion of the enzyme mnk-2 can lead to the development of a new biomarker for testing patient sensitivity to specific drugs.

e) Tumors respond better to PD-1 inhibitors if they first receive radiation or chemotherapy that creates new mutations.

f) Synthetic immune therapies include: Chimeric Antigen Receptors (CAR) or Engineered T-cells.

g) Engineered T-cells (and perhaps also B-cells, another part of the immune system) might treat a wide range of diseases besides cancer: Includes HIV, immune deficiencies, and autoimmune disorders.

h) Engineered cells remain in the body, offering protection against recurrence or re-infection for years to come.

i) In Situ Nano particle V (NP) vaccination: The ability of the immune system to detect and destroy abnormal cells is thought to prevent many cancers; but when tumors start to develop, they can shut down the system, allowing tumors to grow and spread. To restart immune defenses, the tumor itself is used as if it were the antigen in a vaccine, that is, the target for antibodies produced by the immune system. With its infectious components removed, the cowpea mosaic virus (CPMV, a common plant virus) shell acts as the adjuvant, a substance that triggers and may enhance or prolong antigenspecific immune responses. This immunotherapy strategy directly manipulates identified tumors to overcome local tumor-mediated immune suppression and subsequently stimulates systemic anti-tumor immunity to treat metastases. A "something" is placed inside a tumor to disrupt the environment that suppresses the immune system, thus allowing the natural defense system to attack the malignancy. That something, the hard coating of CPMV inhaled into a lung tumor or injected into ovarian, colon or breast tumors, not only triggers the immune system to wipe out the tumors, but provides systemic protection against metastases. Further, being done locally, it does not cause any detectable side effects as is common with traditional therapies and some immune therapies. The CPMV nanoparticles are stable, nontoxic (there are no RNA involved or liposaccharides that may be used as adjuvants), non-irritants (no side effects such as are common with conventional chemotherapy, radiation therapy and with some immuno-stimulation drugs: fatigue, pain, flu-like symptoms, etc.) modifiable with drugs and antigens, and their nano manufacture is highly scalable. These properties, combined with their inherent immunogenicity and demonstrated efficacy against a poorly immunogenic tumor, make them an attractive and novel immunotherapy against metastatic cancer. The particles are shockingly potent, easy to make and do not need to carry antigens, drugs or other immuno-stimulatory agents on their surface or inside. It is not yet clear how the virus shells stimulate the immune system. Unlike most other adjuvants, the virus shells 
stimulate neutrophils, a type of white blood cell whose role is not yet known. In any event, they proved effective against ovarian, breast and colon tumor models. Most of the tumors deteriorated from the center and collapsed. The systemic response prevented or attacked metastatic disease.

\section{In bone marrow (autologous, allogenic) transplantation:}

1. Notwithstanding risks, for refractory leukemias, multiple myelomas, and myelodysplastic syndrome: Diseases known to be inherently resistant to chemotherapy.

2. Extension as an adjuvant therapy for high-risk patients with locally advanced cancer: Lengthens survival but many patients relapse.

\section{In palliative care:}

A. Focus on symptom relief and comfort, not on cure.

\section{Nanotechnology and its Medical Applications}

In 1995, approval of the very first nano therapeutic product (Doxil) is generally viewed as the dawn of nano medicine (NM) for human use. By 2013, the number of approved products had reached 54 in all, with another 150 in various stages of clinical trials. A PubMed search on articles relating to NM shows 7,400 hits over 10 years, of which 1,874 were published in 2013 alone. Similarly, the US patent office database shows 409 patents (since 1976) that were granted in NM, with another 679 applications awaiting approval. However, despite substantial research activity and funding, and notwithstanding the wide use of nanotechnology (NT) in medicine (in vitro diagnostics, in vivo imaging, injectable therapeutics), NT has not yet translated to neither clinical success nor revolutionized treatment paradigms as may have been envisaged earlier. In particular, no molecular machine or nano robot has yet entered clinical trials, although research in these areas is picking up pace.

\section{Nanotechnology}

It must first be noted that biological processes, including ones necessary for life and those that lead to cancer, occur at the nano scale. Thus, in fact, we are composed of a multitude of biological nano-machines (Note: Comparative sizes (in nanometers $[\mathrm{nm}]$ ) of several chemical and biological molecules are: glucose molecule $\sim 1$, antibodies $\sim 10$, viruses $\sim 102$, bacteria $\sim 103$, cancer cells $\sim 104-105$ ). The domains of application of nano devices: nano pores, dendrimers, nano tubes, quantum dots, nano shells, etc, are 10-102). Accordingly, nanotechnology provides researchers with the:

I. Opportunity to study and manipulate macromolecules in real time and during the earliest stages of cancer progression.

II. Rapid and sensitive detection of cancer-related molecules, enabling scientists to detect molecular changes even when they occur only in a small percentage of cells.
III. Potential to generate entirely novel and highly effective therapeutic agents.

Nanotechnology also offers important benefits for diagnosis by providing:

a. New molecular contrast agents and materials to enable an earlier and more accurate initial diagnosis [25].

b. Continual monitoring of cancer patient treatment.

Still further, regarding treatment and clinical outcomes [2629], considering that cancer therapies are currently mostly limited to surgery, radiation and chemotherapy, which risk damage to normal tissues or incomplete eradication of the cancer, nanotechnology offers the means to:

1. Target chemotherapies directly and selectively to cancerous cells and neoplasms.

2. Guide in surgical resection of tumors.

3. Enhance the therapeutic efficacy of radiation-based and other treatment modalities, all of which can add up to a decreased risk to the patient and an increased probability of survival.

\section{Areas of application}

Because of their small size, nano scale materials and devices can interact readily with biomolecules both inside and on the surface of cells with the potential to detect disease and deliver treatment in ways previously unimagined. NPs are promising tools in that they can facilitate research, improve molecular imaging, allow early detection and provide diagnosis, prevention, control, and even treatment of cancer:

Cellular and molecular dynamics: NT offers tools to monitor individual cells and molecules and track their movements in their environment. It enables researchers to study, monitor, and manipulate the multiple systems that can go awry (e.g., in the cancer process). NPs can also be used for intracellular targeting, intracellular drugs, mRNA applications, and gene silencing applications.

Molecular imaging improvement and early pathology detection: NT can help spot cancer in its earliest stages. Detection of biomarkers using NT evidences features that are undetectable through conventional imaging. In addition, photo luminescent nanoparticles may allow oncologists to visually discriminate between cancerous and healthy cells.

Disease prevention and control: Advances driven by the several national and international cancer initiatives, particularly in proteomics and bioinformatics will enable researchers to identify markers of cancer susceptibility and precancerous lesions. NT can then be used to develop devices that indicate when those markers appear in the body and deliver agents to reverse premalignant changes or even kill those cells that have the potential to become malignant. 
Drug delivery and therapeutics: Because of their diverse capabilities, nano scale devices (ND) can contain both targeting and therapeutic agents (in both single and multidrug approaches). They can deliver high drug levels in several situations, including: anticancer drugs at the tumor site that can also increase chemotherapeutic efficacy; cerebrovascular drugs that can penetrate the blood brain barrier (BBB) for treating brain tumors (gliomas, glioblastomas). "Smart" nano therapeutics may provide the ability to "time" the release of any given drug or to deliver multiple drugs sequentially in a timed manner or at several locations in the body.

\section{Nanoparticles Against Cancer}

Cancer cells are notoriously good at becoming resistant to the drugs meant to kill them. One way they do this is by rerouting their signaling networks, specifically those responsible for the cancer cells' growth, proliferation, and survival. To combat this, patients are often given multiple drugs, so-called "rational combination therapy" (RCT), in the hopes of attacking both primary and alternative pathways, the goal being to preemptively block the cancer cell's escape route. Yet, control of the above process when more than one drug will enter a cell, and how much of each will enter, has been a significant challenge that has limited the efficacy of many combination therapies. The reason is that drugs often have very different chemical properties, which cause them to travel to different parts of the body and enter cancer cells at different rates. NPs have come to the rescue [30-33].

\section{Targeting of cancer cells by nanoparticles}

The layer-by-layer NP consists of three components:

a) A core vesicle that is filled with water and has a fatty, double-layered membrane. It is designed so that it can contain both hydrophilic and hydrophobic drugs.

b) A multilayered shell; and

c) An exterior layer that targets the NP to cancer cells and prevents them from being detected by the immune system.

The purpose of the multilayered shell is to stabilize the particles, prevent drug leakage, target the particles to the slightly acidic environment of the tumor, and minimize the particle's interactions with non-cancerous cells. The multilayered shell can also be used to transport drugs that are not easily stored in the core, such as highly charged nucleic acids. These molecules can be spread out within different layers of the shell so that they are separated from drugs that can inactivate their therapeutic effects, for example, platinum drugs. The exterior layer contains molecules that further target the nanoparticles to cancer cells and help them pass through the body unnoticed by the immune system. The above approach may be applicable to several types of cancer since the targeted pathways are present in a variety of tumors, which makes the result particularly significant.

\section{Nanooncology}

The unique characteristics of NPs make them highly attractive for various applications in oncology. They are able to function as:

a. Carriers for chemotherapeutic drugs to increase their therapeutic index and lower their toxicity.

b. Therapeutic agents in photodynamic, gene, and thermal therapy, as well as

c. Molecular imaging agents to detect and monitor cancer progression.

Several NP-based agents for cancer therapy and diagnostics have been approved by the U.S. Food \& Drug Administration (FDA), more are in clinical trials, and even more are in the discovery and early development stages in academic and industry laboratories. Topics of study in nanooncology (NO) also include photodynamic therapy (PDT); targeted photothermal therapy (TPT); thermal ablation of cancer; carriers (liposomal, solid lipid, polymeric, mesoporous silica, and inorganic nanoparticles).

\section{Recent developments}

Visualization: The technique uses luminescent tags, which are quantum dots (QD) attached to proteins that penetrate cell membranes. The dots can be random in size and made of bio-inert material. They can be illuminated by either light of discrete frequency or light encompassing a spectrum of diverse frequencies. Since light color is correlated with particle size, the QDs can be made to fluoresce for a discrete incident light frequency or, equivalently, for a discreet particle size illuminated by several different light frequencies.

However, the bio distribution of these NPs is still imperfect due to the complex host's reactions to nano-and micro-sized materials, and the difficulty in targeting specific organs in the body. Thus, positively-charged gold NPs enter the kidneys while negatively-charged gold NPs remain in the liver and spleen. Even at the small size of $5 \mathrm{~nm}$, these particles can become compartmentalized in the peripheral tissues and will therefore accumulate in the body over time. However, while targeting and distribution can be augmented by NPs, the danger of nanotoxicity becomes an important next step in further understanding their medical uses.

\section{In vivo imaging:}

Nano particle contrast agents: Images such as ultrasound and magnetic resonance imaging (MRI) have generally a favorable distribution and improved contrast and particularly in the case of cancers [25].

Quantum dots: When used in conjunction with MRI, they can produce exceptional images of tumor sites. They are much brighter than organic dyes and only need one light source for 
excitation. "Fluorescent quantum dots" (FQD) could produce a higher contrast image and at a lower cost than today's organic dyes used as contrast media. The downside is that MCs are usually made of quite toxic elements [34]. As an example, QDs of cadmium selenide glow when exposed to UV light. When injected, they seep into cancer tumors. The surgeon can see the glowing tumor, and use it as a guide for more accurate tumor removal.

Multifunctional nanoparticles: Because NPs have high surface area to volume ratio, many functional groups could be attached to them so as to seek and bind to certain tumor cells. Their small size allows them to preferentially accumulate at tumor sites (because tumors lack an effective lymphatic drainage system) and be obliterated by directed RF energy. It is also possible to manufacture multifunctional NPs that would detect, image, and then proceed to treat a tumor.

\section{Drug delivery}

Engineered nanoscale devices: Nanoscale devices can be engineered to aid the delivery of life-saving drug treatments (including cancer) at the affected sites. Such minute devices have the potential to be engineered to efficiently and more safely deliver drug treatments directly to the location of diseased cells while helping avoid harm to healthy cells that fall victim to toxic drugs administered by conventional means.

Bioavailability-improved nanoscale particles and molecules: Nanoscale particles and molecules can also be developed to improve drug bioavailability, i.e., the presence of drug molecules where they are needed in the body and where they will do the most good. Drug delivery focuses on maximizing bioavailability both at specific places in the body and over a period of time. It can be achieved by molecular targeting by nano-engineered devices targeting the molecules and delivering drugs with cell precision. The basic process to use drug delivery involves at least three steps: (i) Encapsulation of the drugs; (ii) Successful delivery of said drugs to the targeted region of the body; and (iii) Successful release of that drug there. Several NPs are employed, including: nutshells (that can be targeted to bond to cancerous cells by conjugated antibodies or peptides to anopheles' surface); platelet-coated NPs (that can deliver higher doses of medication drugs to targeted sites in the body, thus greatly enhancing their therapeutic effects); biocompatible and biodegradable gelatin NPs (that can deliver multiple drugs to the brain bypassing the blood brain barrier (BBB) to treat a variety of brain injuries and neurological diseases in stroke and other victims); and shape-shifting engineered NPs (that can be tailored to deliver drugs to specified tumors and nowhere else).

Development of miniaturized drug delivery systems: Several "nano-carriages" for drug delivery to the right address have been created but many challenges remain, chief among them being how not to let the medicine act before it gets to the right place in the body. The carriers usually encapsulate drugs through long-range electrostatic interactions wherein the carrier attracts oppositely charged medicine. Other tools are available to trigger the release of drugs, for example, an external magnetic field, different $\mathrm{pH}$ values, etc. Such systems, loaded with lifesaving drugs, may revolutionize the way in which cancer is treated with chemotherapy, reducing the debilitating side effects of the therapy, making medications more effective, and all the while preserving the healthy living cells. These include: (a) Protein Cages (containing the anticancer drug daunomycin, a small amount of acid and set at a pH below neutral), which slightly open to let the drug jump inside the tumor, stay in until it came in contact with cancer cells. They can kill more than $70 \%$ without attacking healthy cells; (b) Micro bubbles (microscopic balls of gas enclosed in an ultra-thin layer of fat which can be injected into the blood stream). Theoretically, upon reaching the unhealthy part of the body, they are burst with ultrasound waves, releasing the drug exactly where it is needed. Because the entire blood stream is not being flooded with the drug, side-effects from chemotherapy can be greatly reduced; and (c) Multi-shell hollow nano gels with responsive shell permeability (filled by guest molecules locked in the cavity and released under temperature control). The outer porous shell plays a protective (stabilizing) role and hinders aggregation of the nano-capsules, while the pores of the inner shell can open and close depending on the temperature due to the variable interactions between its monomeric units. At the time of filling, the pores of both shells are open and the nano gel absorbs the drug molecules as a sponge. Then, the temperature changes and the pores of the inner shell close locking in the cavity and readying the drug for delivery. Subsequently, the pores will open again and the guest molecules will be released only in the places where the temperature allows.

There are several clinical advantages to this NPs. Specifically, they:

a) Circulate throughout the bloodstream without being attacked by the immune system.

b) Preferentially bind to cancerous areas allowing them to deliver and release their drug payloads specifically there.

c) Are non-toxic as the platelet membranes are nanoparticle cores made of a biodegradable polymer that can be safely metabolized by the body; and

d) Can be packed with many small drug molecules that diffuse out of the polymer core and through the platelet membrane onto their targets [35-37].

\section{Detection of faint early cancer traces}

Nanoscale magnets offer a new way to find faint early traces of cancer in patients. They make use of magnetic relaxometry signals from iron-oxide nanoparticles that find and attach themselves to cancerous cells. Such cancer signals would never be detected by X-rays. All magnets (or materials prone to magnetism) have magnetic "moments" like invisible needles that can move and react to magnetic fields, even if their physical hosts 
cannot. When exposed to an external magnetic field, these ghostly needles align with it; when the field is removed, they "relax" once again. Relaxometry measures this latter characteristic. This is the very principle of magnetic resonance imaging (MRI). It turns out the moments relax at a very different rate when they belong to nanoparticles that are bound to cancer cells [38].

\section{Treatments of Malignant Tumors}

\section{Virtual biopsy}

Brain tumors can be primary (gliomas, glioblastomas, meningiomas) or secondary resulting from metastasis of other primary tumors (such as lung or breast, radiation necrosis, infections from other organs). On MRI imaging, they produce enhancements whose differential diagnosis remains a significant problem, necessitating an often dangerous if not fatal brain biopsy. Fortunately, a more precise noninvasive MRI diagnostic method has been developed (utilizing a new class of poly ( $\beta$-lmalic acid) polymeric nano imaging tracer agents (NIAs). These agents are able to pass through the BBB and specifically target cancer cells for efficient imaging.

A qualitative/quantitative MRI virtual biopsy method utilizes a nano conjugate-carrying MRI contrast agent gadoliniumDOTA and antibodies that recognize tumor-specific markers and extravasate through the BBB. Using this method, breast and lung cancer brain metastases were successfully treated with tumor-targeted nano conjugates that carried molecular inhibitors of EGFR or HER2 instead of an imaging contrast agent The treatment resulted in a significant increase in survival and markedly reduced immune staining for several cancer stem cell markers. Thus, NIAs are clinically useful for brain diagnostic MRI without currently performing brain biopsies. This technology shows promise for differential MRI diagnosis and treatment of brain metastases and other pathologies when biopsies are difficult to perform.

\section{Brain tumors}

Glioblastoma multiform (GBM), a cancer of the brain also known as "octopus tumors" because of the manner in which the cancer cells extend their tendrils into the surrounding tissue, is virtually inoperable, resistant to therapies, and always fatal, usually within 15 months of onset. Each year, GBM kills approximately 15,000 people in the United States. One of the major obstacles to treatment is the blood brain barrier (BBB), the network of blood vessels that allows essential nutrients to enter the brain but blocks the passage of other substances.

Although there are FDA-approved therapeutic drugs for the treatment of GBM, these treatments have had little impact on patient survival rate because the BBB has limited the accumulation of therapeutics within the brain. What is desperately needed is a means of effectively transporting therapeutic drugs through this barrier. A solution to this dilemma may have been found. It consists of a new family of nano carriers formed from the self- assembly of amphiphilic peptides and polymers, called " $3 \mathrm{HM}$ " for coiled-coil 3-helix micelles. At only $20 \mathrm{~nm}$ in size and featuring a unique hierarchical structure, these new 3HM nano carriers meet all the size and stability requirements for effectively delivering therapeutic drugs to GBM tumors. Amphiphiles are chemical compounds that feature both hydrophilic (waterloving) and lipophilic (fat-loving) properties. Micelles are spherical aggregates of amphiphiles. Using the radioactive form of copper ( $\mathrm{Cu}-64)$ in combination with positron emission tomography (PET) and magnetic resonance imaging (MRI), it has been demonstrated that $3 \mathrm{HM}$ can cross the BBB and accumulate inside GBM tumors at nearly double the concentration rate of current FDA-approved nano carriers [39-47].

\section{Lung cancer and chronic obstructive pulmonary disease}

Nanoparticles can penetrate deep into lung compartments that are otherwise impossible to reach, enabling the treatment of chronic obstructive pulmonary disease (COPD). Commonly, treatment of COPD and lung cancer involves chemotherapeutics and corticosteroids misted into a fine spray that is inhaled for direct delivery to the lungs and quick medicinal effect. However, because the particles produced by today's inhalers are large, most of the medicine is deposited in the upper respiratory tract. "Smart" nanoparticles have been developed to deliver appropriate levels of diagnostic and therapeutic agents to the deepest, tiniest sacs of the lung. (Manipulating immune cells also represents an important strategy for treating lung diseases like COPD and lung cancer, as well as infectious diseases including tuberculosis and listeriosis.)

\section{Pancreatic cancer}

Nanoparticles combine photodynamic and molecular therapies against pancreatic cancer. A nano particle drugdelivery system that combines two complementary types of anticancer treatment could improve outcomes for patients with pancreatic cancer and other highly treatment-resistant tumors while decreasing toxicity. Such a nano medicine approach that combines a drug and photodynamic therapy (PDT) has reduced a thousand-fold the dosage of the molecular therapy drug required to suppress tumor progression and metastatic outgrowth in an animal model [16]. This new optically active nano particle is able both to achieve tumor photo-damage and to suppress multiple escape pathways, opening new possibilities for synchronized multidrug combination therapies and tumor-focused drug release.

\section{Ocular cancer}

A new nano particle that uses a tumor cell's protective mechanism against itself, short-circuiting tumor cell metabolism and killing tumor cells has been developed [17]. It uses a semiconducting nano particle with an attached platinum electrode to drive the synthesis of an anti-cancer compound when illuminated by light. The treatment offers many advantages: 
a. The nano particle produces about 20 million toxins per hour in each cell.

b. It is activated by light so it can be turned on and off simply by exposing it to the correct color of visible light; and

c. It has the potential to be used for multiple applications in ophthalmology and other disciplines.

\section{Chemotherapy}

Tracking the path of chemotherapeutic drugs in real time and at the cellular level could revolutionize cancer care and help sort out why two patients might respond differently to the same treatment. Up until now, this was accomplished, admittedly in a limited way, by organic dyes (that faded quickly) and by toxic elements (particularly, metals). A new organic technique using nanotechnology has been devised to light up the common cancer drug doxoribucin (although any other cancer drug could be employed). Composed of natural amino acids, the nano particle is inherently biocompatible. This work was completed in the laboratory and is currently being tested on animals.

\section{Nano chemotherapy versus Conventional Chemotherapy}

Conventional chemotherapy employs drugs that are known to kill cancer cells effectively. But these cytotoxic drugs kill healthy cells in addition to tumor cells, leading to adverse side effects such as nausea, neuropathy, hair-loss, fatigue, and compromised immune function. On the other hand, NPS can be used as drug carriers for chemotherapeutics delivering medication directly to the tumor while sparing healthy tissue [48]. They can be targeted either passively or actively or in a combination of both. Nano carriers have several advantages over conventional chemotherapy. They can:

a. Protect drugs from being degraded in the body before they reach their target.

b. Enhance the absorption of drugs into tumors and into the cancerous cells themselves.

c. Allow for better control over the timing and distribution of drugs to the tissue, making it easier for oncologists to assess how well they work; and

d. Prevent drugs from interacting with normal cells, thus avoiding side effects.

\section{Passive targeting}

Passive targeting of nano carrier-based drugs relies on a process known as "enhanced permeability and retention". Because of their size and surface properties, certain nanoparticles can escape through blood vessel walls into tissues. In addition, tumors tend to have leaky blood vessels and defective lymphatic drainage, causing NPS to accumulate in them, thereby concentrating the attached cytotoxic drug where it is needed, protecting healthy tissue and greatly reducing adverse side effects.

\section{Active targeting}

Active targeting is based on molecules expressed on their cell surface that bind particular cellular receptors that are attached to a NP. This type of targeting can even be used to bring drugs into the cancerous cell, by inducing the cell to absorb the nano carrier. Active targeting can be combined with passive targeting to further reduce the interaction of carried drugs with healthy tissue. Nanotechnology-enabled active and passive targeting can also increase the efficacy of chemotherapy, achieving greater tumor reduction with lower doses of the drug.

\section{Destruction from within cells}

Moving away from conventional chemotherapeutic agents that activate normal molecular mechanisms to induce cell death, cancerous cells can be physically destroyed from within. One such technology (the nano shells described previously) can thermally destroy tumors from the inside. Nano shells can be designed to absorb light of different frequencies, generating heat (hyperthermia). Once the cancer cells take up the nano shells (via active targeting), near-infrared (NIR) light can be applied creating an intense heat inside the tumor that selectively kills tumor cells without disturbing neighboring healthy cells. Similarly, new targeted magnetic nanoparticles (MNPS, also described above) will both be visible through MRI and cancerous cells can be destroyed by hyperthermia.

\section{New Directions Urged to Improve Cancer Nanotechnology}

Researchers involved in a national effort to develop cancer treatments that harness NT are recommending pivotal changes in the field because experiments with laboratory animals and efforts based on current assumptions about drug delivery have largely failed to translate into successful clinical results. They are trying to perfect "targeted delivery" methods using various agents, including an assortment of tiny nanometersize structures, to selectively attack tumor tissue. However, the current direction of research has brought only limited progress. The bottom line is that so far there are only a few successful NP formulations approved and clinically used. Research with laboratory mice has rarely translated into successful clinical results in humans, suggesting that a more effective approach might be to concentrate on research using in vitro experiments that mimic human physiology. For example, one new system under development, called a "tumor-microenvironment-on-chip (T-MOC)" device, could allow researchers to study the complex environment surrounding tumors and the barriers that prevent the targeted delivery of therapeutic agents. One approach has been to design NPs small enough to pass through pores in blood vessels surrounding tumors but too large to pass though the pores of vessels in healthy tissue. The endothelial cells that make up healthy blood vessels are well organized with tight junctions between them. However, the endothelial cells in blood vessels around tumors are irregular and misshapen, with loose gaps between the cells. 
The approach could help drug makers solve a daunting obstacle: even if drugs are delivered to areas near the target tumor cells, the treatment still is hindered by the complex microenvironment of tumors. It is now known that merely killing the tumor cell would not cure the cancer because the complex environment (non-cancerous cells, blood vessel structure) supports the cancer cells. An "extracellular matrix" near tumors includes dense collagen bundles and a variety of enzymes, growth factors and cells such as, for example, surrounding pancreatic tumors in a "stromal compartment" containing a mixture of cells called stromal cells, activated cancer-associated fibroblasts, and inflammatory immune cells. The stromal tissue is much bigger than the tumor itself. In addition, a compound called hyaluronic acid in this stromal layer increases the toughness of the tumor microenvironment tissue, making it difficult for NPs and drugs to penetrate.

Another challenge is to develop water-soluble drugs to effectively deliver medicines. Indeed, cancer drugs need to be aqueous because the body resorbs them better, but a lot of the current chemotherapy drugs have low solubility and usually need different types of solvents to increase their solubility. The T-MOC approach offers some hope of learning how to design more effective cancer treatments. Recent advances in tissue engineering and micro fluidic technologies present an opportunity to realize in vitro platforms as alternatives to animal testing. However, such a major shift in research focus could play a role in developing personalized medicine (or precision medicine) tailored to a particular type of cancer and specific patients. More effective treatment might require various "priming agents" in combination with several drugs to be administered simultaneously or sequentially. Moreover, since small animal data have not been good predictors of clinical outcome, it is essential to develop in vitro test methods that can represent the microenvironment of human tumors.

\section{Conclusion}

Nanotechnology provides the opportunity to study and manipulate macromolecules in real time and during the earliest stages of cancer progression. It allows rapid and sensitive detection of cancer-related molecules and changes even when they occur in only in a small percentage of cells. It has the potential to generate entirely novel and highly effective therapeutic agents. It offers several important benefits such as the development of new contrast agents for the earlier and more accurate detection of cancer and the continual monitoring of cancer patient treatment. Nanoparticles offer several clinical advantages. They circulate throughout the bloodstream without being attacked by the immune system; they preferentially bind to cancerous areas allowing them to deliver and release their drug payloads specifically there; and they are non-toxic as the platelet membranes are nano particle cores made of a biodegradable polymer that can be safely metabolized by the body; and they can be packed with many small drug molecules that diffuse out of the polymer core and through the platelet membrane onto their targets. They are also able to detect faint early cancer traces.

In contrast with conventional therapies, which risk damage to normal tissues or incomplete eradication of the cancer, nanotechnology offers the means to target chemotherapies directly and selectively to cancerous cells and neoplasms. It can guide in the surgical resection of tumors. It can also enhance the therapeutic efficacy of radiation-based and other treatment modalities, all of which can add up to a decreased risk to the patient and an increased probability of survival.

The unique characteristics of nanoparticles make them highly attractive for various applications in oncology. They are able to function as carriers for chemotherapeutic drugs to increase their therapeutic index and lower their toxicity. They can act as therapeutic agents in photodynamic, gene, and thermal therapy. They also provide imaging agents to detect and monitor cancer progression. Importantly, a variety of miniaturized drug delivery systems have been developed some of which for delivery across the brain protective barriers.

Notwithstanding the general belief that nanoparticles are non-toxic, it remains that their bio distribution is still imperfect due to the complex host's reactions to nano- and micro-sized materials, and also due to the difficulty in targeting specific organs in the body. Thus, even at small sizes, these particles can become compartmentalized in the peripheral tissues and will therefore accumulate in the body over time. While targeting and distribution can be augmented by nanoparticles, the eventual dangers of nanotoxicity become an important next step in further understanding their medical uses.

\section{References}

1. AL Fymat (2016) The Long Quest for Cancer Cures. J Cancer Prev Curr Res 6(2): 00201.

2. Weinberg R (1999) One Renegade Cell: How Cancer Begins (Science Masters). New York, USA.

3. Weinberg R (1997) Racing to the Beginning of the Road: The Search for the Origin of Cancer. Public Health Rep 112(2): 170-171.

4. Weinberg R (2007) The Biology of Cancer. Garland Science 1-73.

5. AL Fymat (2017) Genetics, Epigenetics and Cancer. Cancer Therapy and Oncology International Journal 4(2): 1-11.

6. Jones PA, Baylin SB (2002) The Fundamental Role of Epigenetic Events in Cancer. Nat Rev Genet 3(6): 415-428.

7. Egger G, Liang G, Aparicio A, Jones PA (2004) Epigenetics in Human Disease and Prospects for Epigenetic Therapy. Nature 429(6990): 457463.

8. Feinberg AP, Tycko B (2004) The History of Cancer Epigenetics. Nat Rev Cancer 4(2): 143-153.

9. Novak K (2004) Epigenetic Changes in Cancer Cells. Med Gen Med 6(4): 17.

10. Wong NC, Craig JM (2011) Epigenetics: A Reference Manual. Norfolk, Caister Academic Press, England, pp. 449.

11. Hanahan D, Weinberg R (2001) The Hallmarks of Cancer. Cell 100(1): 57-70. 
12. AL Fymat (2016) Recent Research Developments in Anti-Cancer Therapy. J Cancer Prev Curr Res 5(2): 00155.

13. Lyss AP, Fagundes HM, Corrigan P (2005) Chemotherapy and Radiation for Dummies. Wiley Publishing Inc. New Jersey, USA.

14. Mir LM, Orlowski S, BelehradekJ, Paoletti C (1991) Electrochemotherapy potentiation of antitumour effect of bleomycin by local electric pulses. Eur J Cancer 27(1): 68-72.

15. Mir LM, Belehradek M, Domenge C, Orlowski S, Poddevin B, et al. (1991) Electrochemotherapy, a new antitumor treatment: first clinical trial. C R Acad Sci 313(13): 613-618.

16. Sersa G, Cemazar M, Snoj M (2008) Electochemotherapy of tumours Curr Oncol 16(2): 34-35.

17. Sersa G, Miklavcic D, Cemazar M, Rudolf Z, Pucihar G, et al. (2008) Electrochemotherapy in treatment of tumors. Eur J Surg Oncol 34(2): 232-240.

18. AL Fymat (2017) On Cancer Electro-and Nano-Chemotherapy. J Cancer Prev Curr Res 7(2): 00232

19. Wang L, Wang W, Rui Z, Zhou D (2016) The effective Combination Therapy Against Human Osteosarcoma: Doxorubicin plus Curcumin Co-Encapsulated Lipid-Coated Polymeric Nanoparticulate Drug Delivery System, Drug Deliv 23(9): 3200-3208.

20. Maude SL, Frey N, Shaw PA, Aplenc R, Barrett DM, et al. (2014) Chimeric Antigen Receptors T-Cells for Sustained Remissions in Leukemia. N Engl J Med 371(16): 1507-1517.

21. Maus MV, Fraietta JA, Levine BL, Kalos M, Zhao Y, et al. (2014) Adoptive Immunotherapy for Cancer or Viruses. Annu Rev Immunol 32: 189225

22. Posey AD, June CH, Levine BL (2017) Cancer Killers. Sci Am 316(3): 38-43.

23. AL Fymat (2017) Immunotherapy: An Emergent Anti-Cancer Strategy. J Cancer Prev Curr Res 7(3): 00233.

24. AL Fymat (2017) Synthetic Immunotherapy with Chimeric Antigen Receptors. J Cancer Prev Curr Res 7(5): 00253.

25. AL Fymat (2017) Magnetic Resonance Imaging Modalities with Nano contrasting Materials. J Curr Trends Clin Med Imaging 1(1): 555554.

26. Grodzinski P, Farrell D, Hinkal G, Hook SS, Panaro N, et al. (2010) Cancer Nanotechnology Plan. National Cancer Institute, USA, p. 1-84.

27. Nanotech News: An online resource for news about cancer nanotechnology research, England.

28. (2004) NCI Alliance for Nanotechnology in Cancer, National Institute of Health, p. 1-2.

29. AL Fymat (2016) Nanotechnology and Cancer. J Cancer Prev Curr Res 5(6): 00180.

30. (2004) Alliance for Nanotechnology in Cancer. U.S. National Cancer Institute, USA.
31. (2003) Scientific Forward Look on Nano medicine. European Science Foundation p. 1-52.

32. Roadmap (now Common Fund) of the Nanomedicine Initiative, U.S. National Institute of Health, USA.

33. AL Fymat (2016) Recent Developments in Nanomedicine Research. J Nanomed Res 4(4): 00096.

34. Simak J (2016) Investigation of Potential Toxic Effects of Engineered Nanoparticles and Biologic Microparticles in Blood and Their Biomarker Applications. U.S. Food and Drug Administration.

35. Nie S, Xing Y, Kim GJ, Simons JW (2007) Nanotechnology Applications in Cancer. Annu Rev Biomed Eng 9: 257-288.

36. Ashton S, Song YH, Nolan J, Cadogan E, Murray J, et al. (2016) Aurora kinase inhibitor nanoparticles target tumors with favorable therapeutic index in vivo. Science Translational Medicine 8(325): 325Ra17.

37. Jain KK (2007) Nanobiotechnology-Based Drug Delivery to the Central Nervous System. Neurodegener Dis 4(4): 287-291.

38. Rivière B (2016) Magnetic Nanoparticles Can Detect Early Traces of Cancer. RICE.

39. Jolliet-Riant P, Tillemont JP (1999) Drug Transfer Across the BloodBrain Barrier and Improvement of Brain Delivery. Fundam Clin Pharmacol 13(1): 16-26.

40. Temsamani J, Rousselle C, Reese AR, Scherrmann JM (2001) Vectormediated Drug Delivery to the Brain. Expert Opin Biol Ther 1(5): 773782.

41. Scherrmann JM (2002) Drug Delivery to Brain via the Blood-Brain Barrier. Vascul Pharmacol 38(6): 349-354.

42. Lockman PR, Mumper RJ, Khan MA, Allen DD (2002) Nanoparticle Technology for Drug Delivery across the Blood-Brain Barrier. Drug Dev Ind Pharm 28(1): 1-13.

43. Chen Y, Dalwadi G, Benson HA (2004) Drug Delivery across the BloodBrain Barrier. Curr Drug Deliv 1(4): 361-376.

44. Patel MM, Goyal BR, Bhadada S, Bhatt JS, Amin AF (2009) Getting into the Brain: Approaches to Enhance Brain Drug Delivery. CNS Drugs 23(1): 35-58.

45. Weiss N, Miller F, Cazaubon S, Couraud PO (2010) Blood-brain Barrier part III: Therapeutic Approaches to Cross the Blood-Brain Barrier and Target the Brain. Rev Neurol (Paris) 166(3): 284-288.

46. Dinda SC, Pattnaik G (2013) Nanobiotechnology-Based Drug Delivery in Brain Targeting. Curr Pharm Biotechnol 14(15): 1264-1274.

47. AL Fymat (2017) Nanoneurology: Drug Delivery across the Brain Protective Barriers. J Nanomed Res 5(1): 00105.

48. AL Fymat (2017) Nanochemotherapy: An Emergent Anti-Cancer Modality. J Nanomed Res 1(1): 1-6.

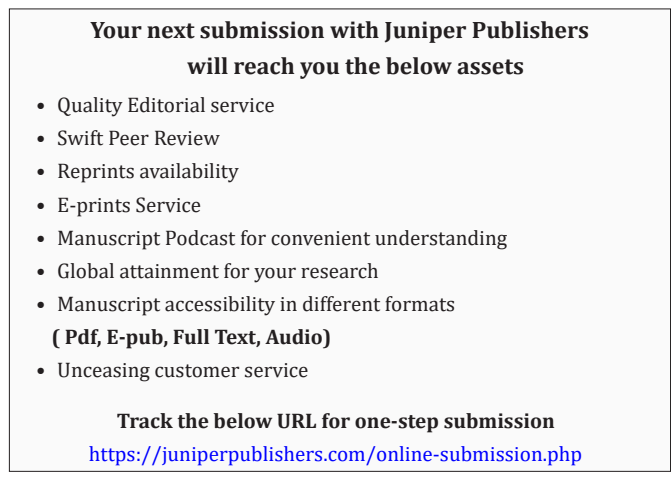

